Check for updates

Cite this: Chem. Sci., 2018, 9, 6260

๑ All publication charges for this article have been paid for by the Royal Society of Chemistry

Received 24th April 2018

Accepted 28th June 2018

DOI: $10.1039 / \mathrm{c} 8 \mathrm{sc} 01869 \mathrm{c}$

rsc.li/chemical-science

\title{
Enantioselective synthesis of 2-oxazolidinones by ruthenium(II)-NHC-catalysed asymmetric hydrogenation of 2-oxazolones $\uparrow$
}

\begin{abstract}
Wei Li, (D) Marco Wollenburg and Frank Glorius (D)*
An efficient synthesis of optically active 4-substituted 2-oxazolidinones by the ruthenium(॥)-NHCcatalysed asymmetric hydrogenation of 2-oxazolones was developed. Excellent enantioselectivities (up to $96 \%$ ee) and yields (up to $99 \%$ ) were obtained for a variety of substrates, bearing a range of functional groups and useful motifs. The hydrogenation reaction was successfully scaled up to gram scale using low catalyst loading. Moreover, the utility of this methodology was demonstrated by the transformation of the enantioenriched product into the corresponding chiral $\beta$-amino alcohol, a bisoxazoline ligand, and the formal synthesis of (-)-aurantioclavine.
\end{abstract}

\section{Introduction}

Chiral 2-oxazolidinones, widely used as Evans' chiral auxiliaries (Scheme 1a, left), play a prominent role in modern organic synthesis. ${ }^{1}$ Based on chiral 2-oxazolidinone auxiliaries, a wide range of asymmetric transformations has been developed to construct new chiral building blocks, which are frequently used in both natural product synthesis and drug discovery. ${ }^{2}$ a)

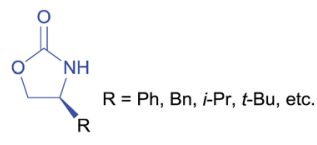

Evans' chiral auxiliaries

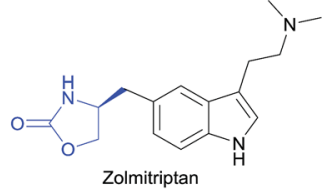

Zolmitriptan b) Conventional approach (preformed stereocenter)

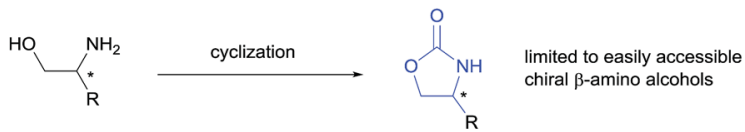

c) Alternative strategy (late-stage introduction of the stereocenter)

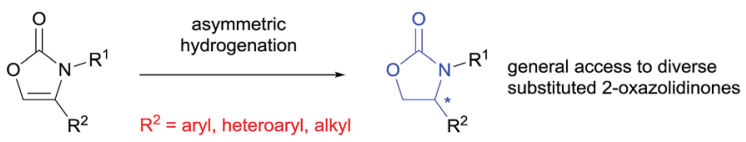

Scheme 1 Applications and synthesis of chiral 2-oxazolidinones.

Organisch-Chemisches Institut, Westfälische Wilhelms-Universität Münster, Corrensstraße 40, 48149 Münster, Germany.E-mail: glorius@uni-muenster.de $\dagger$ Electronic supplementary information (ESI) available. CCDC 1584965. For ESI and crystallographic data in CIF or other electronic format see DOI: $10.1039 / \mathrm{c} 8 \mathrm{sc} 01869 \mathrm{c}$
Furthermore, the chiral 2-oxazolidinone motif itself is common in pharmaceutically relevant molecules (Scheme 1a, right). ${ }^{3}$ Thus, the synthesis of chiral 2-oxazolidinones has already attracted considerable attention. Conventionally, enantioenriched 2-oxazolidinones are synthesized by cyclization of the corresponding optically pure $\beta$-amino alcohols with C1building blocks like phosgene and its derivatives (Scheme 1b). These methods often require toxic reagents and the preconstruction of the key stereocenter of the $\beta$-amino alcohols arises synthetic problems if they cannot be formed from natural enantiopure amino acids or related precursors. ${ }^{2}$ Considering this, the exploration of orthogonal, efficient and divergent catalytic strategies for the construction of diverse 2-oxazolidinone derivatives is highly important for organic synthesis and drug discovery.

In the last decade, the asymmetric hydrogenation of unsaturated heterocycles has emerged as a conceptually powerful method to produce optically active cyclic compounds and has received significant attention. ${ }^{4}$ In this regard, a synthetic method utilizing the enantioselective hydrogenation of 2-oxazolones for the late-stage construction of the key stereocenter can be envisioned as a powerful alternative to prepare diverse optically active 2-oxazolidinones (Scheme 1c). Moreover, this strategy would also provide a general way to produce optically active $\beta$-amino alcohols since the transformation from 2-oxazolidinones to free $\beta$-amino alcohols is very convenient. ${ }^{2}$ Recently, Zhang and co-workers reported the rhodium-catalysed asymmetric hydrogenation of 2-oxazolones, which afforded 4aryl substituted 2-oxazolidinones with moderate enantioselectivities. ${ }^{5}$ To the best of our knowledge, this is the only precedent of an enantioselective synthesis of chiral 2-oxazolidinones by asymmetric hydrogenation of unsaturated heterocycles. As a continuous effort in the field of arene and heterocycle hydrogenation, ${ }^{6}$ we herein describe a highly 
enantioselective and practical hydrogenation of a broad scope of 2-oxazolones to access diverse enantioenriched 2-oxazolidinones catalysed by a ruthenium(II)-N-heterocyclic carbene (NHC) complex.

\section{Results and discussion}

Initially, the hydrogenation of 4-phenyloxazol-2(3H)-one (1a) was attempted under 50 bar $\mathrm{H}_{2}$ in $n$-hexane at room temperature in the presence of our previously-developed ruthenium(II)NHC catalyst, which is prepared in situ from $[\mathrm{Ru}(2-$ methylallyl $\left.)_{2}(\mathrm{COD})\right]$, the NHC precursor $(R, R)$-SINpEt $\cdot \mathrm{HBF}_{4}$, and $\mathrm{NaO} t-\mathrm{Bu} .^{7,8}$ However, the desired product was not observed, presumably due to catalyst deactivation by coordination of the metal with free $\mathrm{N}-\mathrm{H}$ (Table 1 , entry 1 ). To circumvent catalyst deactivation, a variety of protecting groups were investigated, of which the 4-methoxybenzyl (PMB) protected carbamate 1d was found to be suitable for hydrogenation, providing the desired 4phenyloxazolidin-2-one with $85 \%$ ee and in $95 \%$ isolated yield (entry 2-4). To further improve the reaction conditions, a solvent screen was conducted (entries 4-8). No reaction occurred in dichloromethane presumably due to catalyst decomposition (entry 5), and cyclohexane (entry 8) was found to be slightly superior to other solvents ( $n$-hexane, toluene and THF) for enantioselectivity. Decreasing the reaction temperature to $0{ }^{\circ} \mathrm{C}$ further improved enantiocontrol, providing the desired product with $95 \%$ ee and in $93 \%$ yield (entry 9 ). Finally, a solvent mixture of cyclohexane/THF $=20 / 1$ was used to

Table 1 Optimisation of the reaction conditions ${ }^{a}$

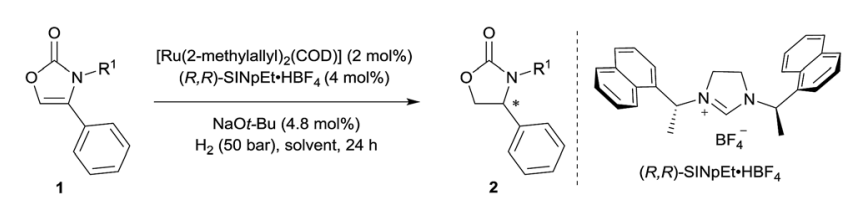

\begin{tabular}{llllll}
\hline Entry & $\mathrm{R}^{1}$ & Solvent & $T\left({ }^{\circ} \mathrm{C}\right)$ & Yield $^{b}(\%)$ & $e e^{c}(\%)$ \\
\hline 1 & H (1a) & $n$-Hexane & 25 & 0 & - \\
2 & Boc (1b) & $n$-Hexane & 25 & Traces & N. D. \\
3 & Ac (1c) & $n$-Hexane & 25 & 0 & - \\
4 & PMB (1d) & $n$-Hexane & 25 & 95 & 85 \\
5 & PMB (1d) & CH $_{2} \mathrm{Cl}_{2}$ & 25 & 0 & - \\
6 & PMB (1d) & Toluene & 25 & 99 & 85 \\
7 & PMB (1d) & THF & 25 & 99 & 87 \\
8 & PMB (1d) & Cyclohexane & 25 & 98 & 88 \\
9 & PMB (1d) & Cyclohexane & 0 & 93 & 95 \\
$10^{d}$ & PMB (1d) & Cyclohexane & 0 & 99 & 95
\end{tabular}

${ }^{a}$ General conditions: [Ru(2-methylallyl $\left.)_{2}(\mathrm{COD})\right]$ (0.10 $\left.\mathrm{mmol}\right),(R, R)$ SINpEt $\cdot \mathrm{HBF}_{4}(0.20 \mathrm{mmol})$ and $\mathrm{NaO} t$-Bu $(0.24 \mathrm{mmol})$ were stirred at $70{ }^{\circ} \mathrm{C}$ in $n$-hexane $(5.0 \mathrm{~mL})$ for $16 \mathrm{~h}$ to perform the catalyst $(0.02 \mathrm{M})$, after which $0.1 \mathrm{~mL}$ of the catalyst suspension were added to substrates 1a-d $(0.10 \mathrm{mmol})$ in the indicated solvent $(1.0 \mathrm{~mL})$, and the hydrogenation was performed at 50 bar $\mathrm{H}_{2}$ for $24 \mathrm{~h} .{ }^{b}$ Yields of isolated product after column chromatography are reported. ${ }^{c}$ Determined by HPLC analysis using a chiral stationary phase. ${ }^{d}$ Using a solvent mixture of cyclohexane $/ \mathrm{THF}=20 / 1$. Boc $=$ tert-butyloxycarbonyl, Ac $=$ acetyl, PMB $=4$-methoxybenzyl. N. D. = not determined. improve the solubility of the substrate, to afford the chiral 2oxazolidinone $2 \mathbf{d}$ in $99 \%$ yield and $95 \%$ ee (entry 10 ).

With the optimised reaction conditions in hand (Table 1, entry 10), the substrate scope of the reaction was explored (Schemes 2

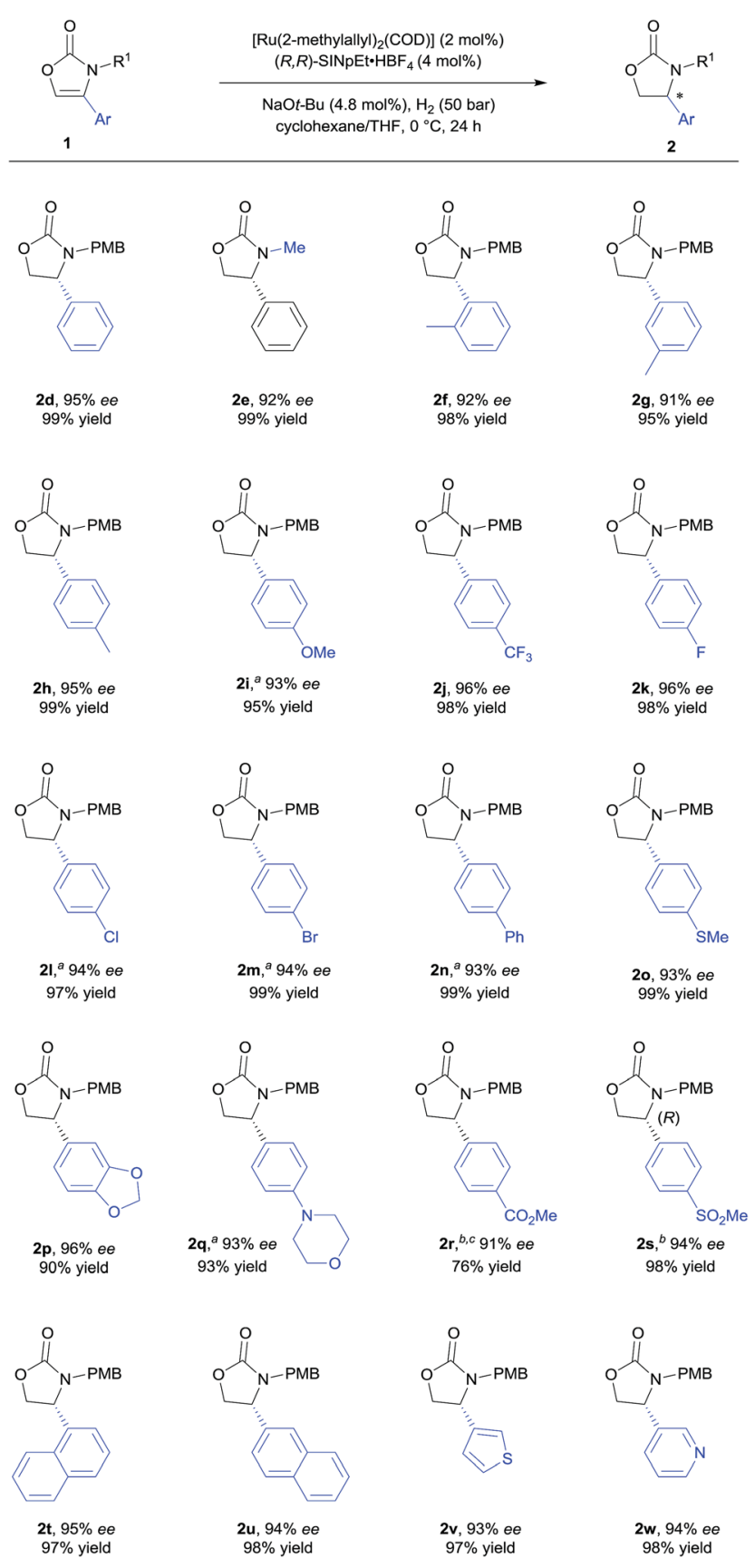

Scheme 2 Substrate scope of 4-aryl or 4-heteroaryl substituted 2oxazolones. General conditions: [Ru(2-methylallyl) $\left.)_{2}(\mathrm{COD})\right] \quad(0.10$ $\mathrm{mmol}),(R, R)-\mathrm{SINpEt} \cdot \mathrm{HBF}_{4}(0.20 \mathrm{mmol})$ and $\mathrm{NaOt}-\mathrm{Bu}(0.24 \mathrm{mmol})$ were stirred at $70{ }^{\circ} \mathrm{C}$ in $n$-hexane $(5.0 \mathrm{~mL})$ for $16 \mathrm{~h}$ to perform the catalyst $(0.02 \mathrm{M})$, after which $0.2 \mathrm{~mL}$ of the catalyst suspension were added to substrates $1 \mathrm{~d}-\mathrm{s}(0.20 \mathrm{mmol})$ in cyclohexane/THF (20/1), and the hydrogenation was performed under 50 bar $\mathrm{H}_{2}$ at $0{ }^{\circ} \mathrm{C}$ for $24 \mathrm{~h}$. Yields of isolated products after column chromatography are reported. $\%$ ee values were determined by HPLC analysis using a chiral stationary phase. ${ }^{a}$ Using a solvent mixture of cyclohexane/THF (1/1). ${ }^{b}$ Using THF (2.0 mL). ${ }^{\circ} \mathrm{At}-10^{\circ} \mathrm{C}$. 
and 3). First, the variation of the protecting group from $N$-PMB to $N$-methyl afforded the products with similar results (Scheme 2, 2d and 2e). Next, the positional influence of substituents on the phenyl ring was investigated. Methyl groups in the 2-, 3- and 4positions were well-tolerated, providing the corresponding 2oxazolidinones with excellent enantioselectivities and in high yields (2f-h). The electronic effect of the substituents was also examined. Both, electron-rich and electron-deficient substrates (1i and $\mathbf{1 j}$ respectively) underwent hydrogenation to smoothly afford products $2 \mathbf{i}$ and $\mathbf{2 j}$. Halogenated substrates $\mathbf{1} \mathbf{k}-\mathbf{m}$ were also used to provide the desired products $2 \mathbf{k}-\mathbf{m}$ with excellent enantioselectivities, in very high yields and without the formation of dehalogenated byproducts. Notably, the catalytic system showcased a robust reactivity, tolerating various functional groups and useful motifs (such as SMe, 1,3-benzodioxole, morpholine, $\mathrm{CO}_{2} \mathrm{Me}$ and $\mathrm{SO}_{2} \mathrm{Me}$ ) to provide the corresponding products $2 \mathbf{0}-\mathbf{S}$ with $91-96 \% e e$ and in 76-99\% yield. These functional groups and motifs (2i-s) provide an excellent opportunity for further applications of the 2-oxazolidinone products. In addition, the absolute configuration of $2 \mathrm{~s}$ was determined to be $(R)$ by X-ray crystallographic analysis. ${ }^{9}$ The absolute configuration of all other products was assigned by analogy. Additionally, substrates with condensed-ring and heteroaromatic moieties were also tested. Both 1- and 2-naphthyl-substituted substrates were tolerated under the standard conditions (2t and $\mathbf{2 u}$ ). Remarkably, thiophene and pyridine containing substrates did not poison the $\mathrm{Ru}-$ NHC catalyst, producing the corresponding products ( $2 \mathbf{v}$ and $2 \mathbf{w}$ ) with $93 \% e e$ and $94 \% e e$ respectively.

We further explored the substrate scope with 4-alkyl substituents (Scheme 3). Alkyl substituted substrates with different steric demand were systematically tested. Simple 4methyloxazolidin-2-one $2 \mathbf{x}$ was obtained with moderate enantioselectivity. Better control of the stereoselectivity was observed
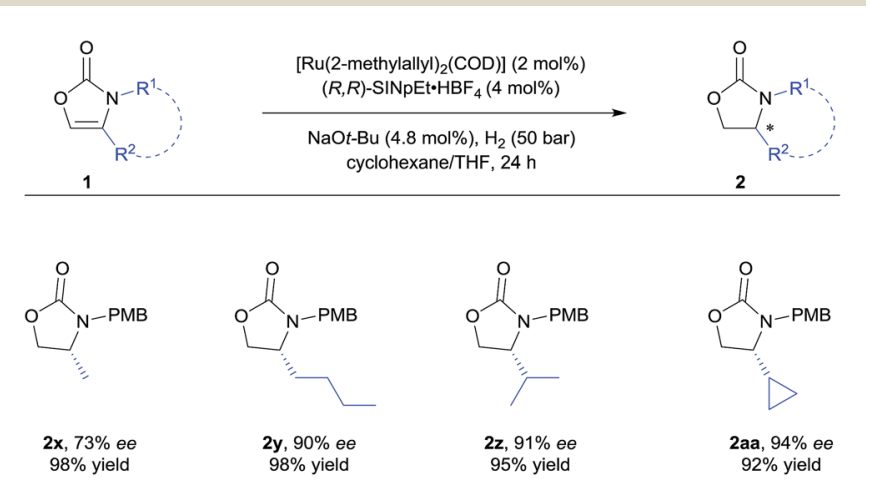

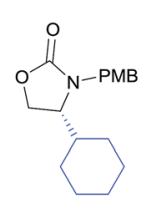

2ab, $94 \%$ ee
$94 \%$ yield
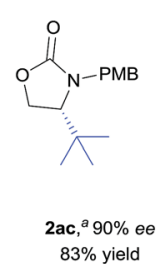
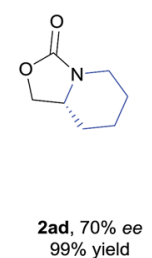

Scheme 3 Substrate scope of 4-alkyl substituted 2-oxazolones. For detailed conditions, see ESI. $\uparrow$ Yields of isolated products after column chromatography are reported. \% ee values were determined by HPLC analysis using a chiral stationary phase. ${ }^{a}$ Using 5 mol\% of the catalyst.

when introducing $n$-butyl substituent (2y). Isopropyl, cyclopropyl, and cyclohexyl substituents were successfully employed, affording the corresponding products with $91-94 \%$ ee and in 92-95\% yield (2z-ab). Finally, tert-butyl substituted 2-oxazolone 1ac was tolerated to give the product 2ac with $90 \%$ ee and in $83 \%$ yield. These results indicate that bulky alkyl groups are beneficial for the enantioinduction. In addition, bicyclic 2oxazolidinone 2ad was also obtained by the developed method, albeit with moderate $e e$.

We then proceeded to demonstrate applications of this methodology (Scheme 4). Scale-up of the hydrogenation of 4-(1naphthyl) substituted substrate 1t to gram-scale provided the corresponding 2-oxazolidinone 2 t with $95 \% e e$ and in $99 \%$ yield (Scheme 4a). Remarkably, the catalyst loading was successfully reduced to $0.2 \mathrm{~mol} \%$, which greatly increases the synthetic viability of this protocol. The deprotection of the PMB group was conveniently completed to afford 3 in $99 \%$ yield and without loss of enantiomeric excess. ${ }^{6 \boldsymbol{d}}$ Hydrolysis of $2 \mathrm{t}$ using $\mathrm{NaOH}$ liberated the $N$-PMB $\beta$-amino alcohol 4 in $92 \%$ yield. The enantiopurity of product 3 was readily increased to $>99 \% e e$ after recrystallization from ethyl acetate. Optically pure $\beta$-amino alcohol $\mathbf{5}$ was then prepared in quantitative yield by cleavage of the 2-oxazolidinone 3 using diethylenetriamine (Scheme $4 \mathrm{~b}$ ). ${ }^{\mathbf{1 0}}$ The absolute configuration of $\beta$-amino alcohol 5 was reaffirmed to be $(R)$ by comparing the optical rotation to the literature data. ${ }^{11}$ The $\beta$-amino alcohol 5 was further transformed into 1naphthyl-substituted bisoxazoline ligand 6 in 74\% yield by reaction with dimethylmalononitrile and $\mathrm{Zn}(\mathrm{OTf})_{2} \cdot{ }^{\mathbf{1 1 a}, \mathbf{1 2}}$ Furthermore, aryl iodide and different $N$-alkyl substituted substrate 1ae was well tolerated under the established reaction conditions (Scheme 4c). Hydrogenation of 1ae using $(S, S)$ -

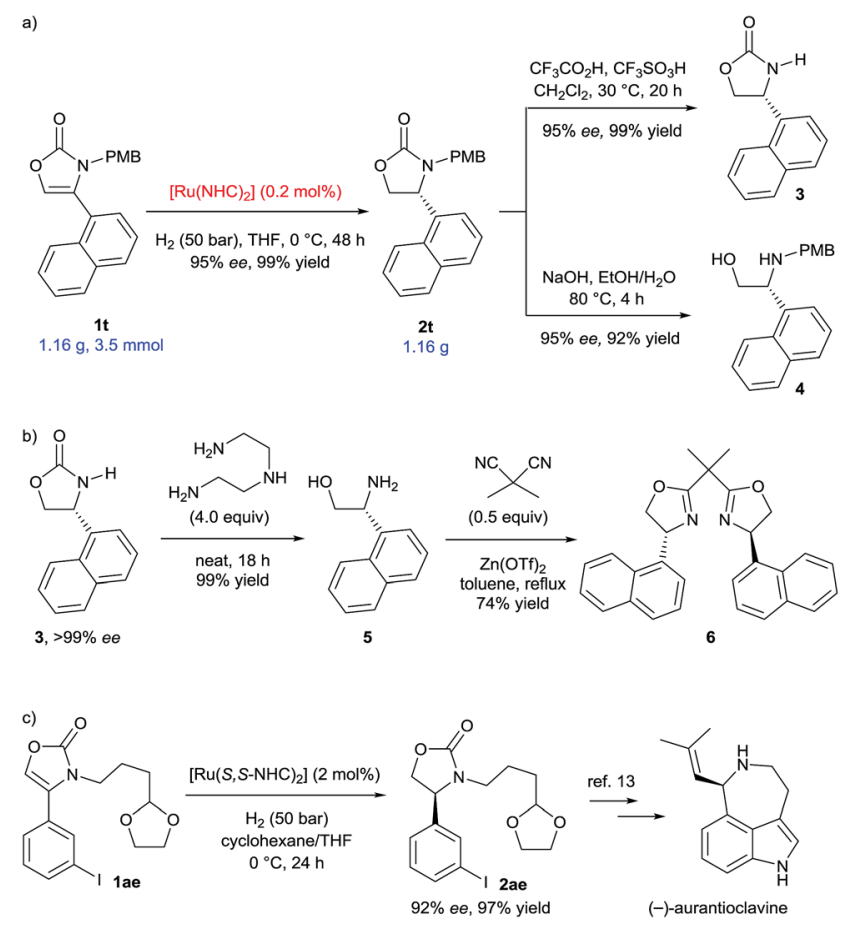

Scheme 4 Scaled-up hydrogenation and transformations of the products. 
SINpEt $\cdot \mathrm{HBF}_{4}$ as the carbene ligand precursor, furnished the oxazolidinone 2 ae ( $92 \%$ ee and $96 \%$ yield), a key synthetic intermediate employed in the synthesis of the alkaloid (-)-aurantioclavine. ${ }^{13}$

\section{Conclusions}

In summary, we have developed a protocol for the catalytic enantioselective hydrogenation of 2-oxazolones to obtain optically active 2-oxazolidinone derivatives. The ruthenium(II)-NHC catalyst system enabled a broad range of substrates to be successfully hydrogenated with excellent enantioselectivities (up to $96 \% e e$ ) and in yields (up to 99\%), thus to make the approach practical for the first time. Various functional groups and synthetically useful motifs were well-tolerated. The synthetic utility of this protocol was further demonstrated by performing a reaction on a gramscale with a reduced catalyst loading; the obtained enantioenriched product was readily converted into an optically pure $\beta$ amino alcohol and subsequently a bisoxazoline ligand. The formal synthesis of (-)-aurantioclavine was enabled by the functional group tolerance towards an iodine substituent at the aryl ring and a varied $N$-alkyl chain.

\section{Conflicts of interest}

There are no conflicts to declare.

\section{Acknowledgements}

We thank the Alexander von Humboldt Foundation (W. L.) and the Deutsche Forschungsgemeinschaft (IRTG 2027, Leibniz Award) for generous support. We thank Mario P. Wiesenfeldt, Christoph Schlepphorst and Dr Michael J. James for many helpful discussions. We thank Dr Constantin G. Daniliuc and Birgit Wibbeling for the X-ray crystallographic analysis.

\section{Notes and references}

1 (a) D. A. Evans, J. Bartroli and T. L. Shih, J. Am. Chem. Soc., 1981, 103, 2127; (b) D. A. Evans, Aldrichimica Acta, 1982, 15, 23.

2 For selected reviews, see: (a) J. Seyden-Penne, Chiral Auxiliaries and Ligands in Asymmetric Synthesis, Wiley, New York, 1995; (b) D. J. Ager, I. Prakash and D. R. Schaad, Chem. Rev., 1996, 96, 835; (c) D. J. Ager, I. Prakash and D. R. Schaad, Aldrichimica Acta, 1997, 30, 3; (d) Catalytic Asymmetric Synthesis, ed. I. Ojima, Wiley, New York, 2000.

3 (a) M. E. Dyen and D. Swern, Chem. Rev., 1967, 67, 197; (b) E. A. MacGregor, Drugs Today, 1998, 34, 1027; (c) A. M. Rapoport, M. E. Bigal, S. J. Tepper and F. D. Sheftell, Expert Rev. Neurother., 2004, 4, 33.

4 For selected reviews, see: (a) W. Tang and X. Zhang, Chem. Rev., 2003, 103, 3029; (b) Y.-G. Zhou, Acc. Chem. Res., 2007, 40, 1357; (c) R. Kuwano, Heterocycles, 2008, 76, 909; (d) J.-H. Xie, S.-F. Zhu and Q.-L. Zhou, Chem. Rev., 2011, 111, 1713; (e) D.-S. Wang, Q.-A. Chen, S.-M. Lu and Y.-G. Zhou, Chem. Rev., 2012, 112, 2557; (f) Z. Yu, W. Jin and Q. Jiang,
Angew. Chem., Int. Ed., 2012, 51, 6060; (g) Y.-M. He, F.-T. Song and Q.-H. Fan, Top. Curr. Chem., 2014, 343, 145; (h) Z. Zhang, N. A. Buttand and W. Zhang, Chem. Rev., 2016, 116, 14769; (i) Z.-P. Chen and Y.-G. Zhou, Synthesis, 2016, 48, 1769.

5 Q. Wang, X. Tan, Z. Zhu, X.-Q. Dong and X. Zhang, Tetrahedron Lett., 2016, 57, 658.

6 (a) S. Urban, N. Ortega and F. Glorius, Angew. Chem., Int. Ed., 2011, 50, 3803; (b) S. Urban, B. Beiring, N. Ortega, D. Paul and F. Glorius, J. Am. Chem. Soc., 2012, 134, 15241; (c) N. Ortega, S. Urban, B. Beiring and F. Glorius, Angew. Chem., Int. Ed., 2012, 51, 1710; (d) W. Li, C. Schlepphorst, C. Daniliuc and F. Glorius, Angew. Chem., Int. Ed., 2016, 55, 3300; (e) W. Li, M. P. Wiesenfeldt and F. Glorius, J. Am. Chem. Soc., 2017, 139, 2585; $(f)$ M. P. Wiesenfeldt, Z. Nairoukh, W. Li and F. Glorius, Science, 2017, 357, 908.

7 Selected reviews for the use of NHCs as ligands in transitionmetal catalysis, see: (a) W. A. Herrmann, Angew. Chem., Int. Ed., 2002, 41, 1290; (b) S. P. Nolan, N-Heterocyclic Carbenes in Synthesis, Wiley-VCH, Weinheim, Germany, 2006; (c) F. Glorius, N-Heterocyclic Carbenes in Transition Metal Catalysis, Springer, Berlin, 2007; (d) V. Dragutan, I. Dragutan, L. Delaude and A. Demonceau, Coord. Chem. Rev., 2007, 251, 765; (e) F. E. Hahn and M. C. Jahnke, Angew. Chem., Int. Ed., 2008, 47, 3122; (f) S. Díez-González, N. Marion and S. P. Nolan, Chem. Rev., 2009, 109, 3612; $(g)$ S. Gaillard, C. S. J. Cazin and S. P. Nolan, Acc. Chem. Res., 2012, 45, 778; (h) C. Valente, S. Çalimsiz, K. H. Hoi, D. Mallik, M. Sayah and M. G. Organ, Angew. Chem., Int. Ed., 2012, 51, 3314; (i) M. N. Hopkinson, C. Richter, M. Schedler and F. Glorius, Nature, 2014, 510, 485.

8 Selected reviews for the use of NHCs as ligands in asymmetric catalysis, see: (a) M. C. Perry and K. Burgess, Tetrahedron: Asymmetry, 2003, 14, 951; (b) V. César, S. Bellemin-Laponnaz and L. H. Gade, Chem. Soc. Rev., 2004, 33, 619; (c) L. H. Gade and S. Bellemin-Laponnaz, Coord. Chem. Rev., 2007, 251, 718; (d) F. Wang, L.-J. Liu, W. Wang, S. Li and M. Shi, Coord. Chem. Rev., 2012, 256, 804; (e) D. Zhao, L. Candish, D. Paul and F. Glorius, ACS Catal., 2016, 6, 5978.

9 CCDC 1584965 contains the supplementary crystallographic data for this paper. $\dagger$

10 M. Noshita, Y. Shimizu, H. Morimoto and T. Ohshima, Org. Lett., 2016, 18, 6062.

11 (a) H. L. van Lingen, J. K. W. van de Mortel, K. F. W. Hekking, F. L. van Delft, T. Sonke and F. P. J. T. Rutjes, Eur. J. Org. Chem., 2003, 317; (b) J. M. Takacs, M. R. Jaber and A. S. Vellekoop, J. Org. Chem., 1998, 63, 2742.

12 (a) A. Cornejo, J. M. Fraile, J. I. García, M. J. Gil, V. MartínezMerino, J. A. Mayoral, E. Pires and I. Villalba, Synlett, 2005, 2321; (b) Z. Zhou and M. B. Andrus, Tetrahedron Lett., 2012, 53, 4518.

13 J. Park, D.-H. Kim, T. Das and C.-G. Cho, Org. Lett., 2016, 18, 5098. 